\title{
Bounds for the Zeros of Polynomials
}

\section{Roshan Lal}

\section{Department of Mathematics}

Govt. Degree College

Chaubattakhal (Pauri Garhwal)

Uttrakhand

rlkeshtwal@yahoo.co.in

Abstract: Let $p(z)$ be a polynomial of degree $n, \quad p(z)=\sum_{v=0}^{n} a_{v} z^{v} \quad$ and $\quad$ also let $\operatorname{Re}\left(a_{j}\right)=\alpha_{j}, \operatorname{Im}\left(a_{j}\right)=\beta_{j}$. In this paper we have obtained a zero-free region in terms of $\alpha_{j}$ and $\beta_{j}$, and also obtained the number of zeros that can lie in a prescribed region. Our result sharpens as well as generalizes the earlier known results.

Keywords: Polynomials; Zeros; Inequalities; Complex domain.

\section{AMS Subject Classification: - 30C15}

\section{1-Introduction and Statement of Results}

The following results are well known in the theory of the distribution of zeros of polynomial.

Theorem A: - If $p(z)=\sum_{v=0}^{n} a_{v} z^{v}$ be a polynomial of degree $n$ with the coefficients satisfying the condition

$$
a_{n} \geq a_{n-1} \geq a_{n-2} \geq \cdots \cdots a_{1} \geq a_{0}>0,
$$

then all zeros of $p(z)$ lie in $|z| \leq 1$.

This is known as Enestr $\ddot{O}$ m-Kakeya theorem $[2,4]$.

Theorem B: - If $p(z)=\sum_{v=0}^{n} a_{v} z^{v}$ be a polynomial of degree $n$. If $a_{j}=\alpha_{j}+i \beta_{j}$ and $\operatorname{Re}\left(a_{k}\right)=\alpha_{k}, \operatorname{Im}\left(a_{k}\right)=\beta_{k}$ for $k=0,1,2, \ldots \ldots, n$ and

$$
\alpha_{n} \geq \alpha_{n-1} \geq \alpha_{n-2} \geq \cdots \cdots \geq \alpha_{1} \geq \alpha_{0} \geq 0, \quad \text { with } \alpha_{n}>0,
$$

then $p(z)$ has all its zeros in the ring-shaped region given by

$$
\frac{\left|a_{0}\right|}{R_{1}^{n-1}\left[2 R_{1} \alpha_{n}+R_{1}\left|\beta_{n}\right|-\left(\alpha_{0}+\left|\beta_{0}\right|\right)\right]} \leq|z| \leq R_{1}=1+\frac{1}{\alpha_{n}}\left[2 \sum_{k=0}^{n-1}\left|\beta_{k}\right|+\left|\beta_{n}\right|\right]
$$

The above result is due to Govil and Rahman [3].

Theorem C: - If $p(z)=\sum_{v=0}^{n} a_{v} z^{v}$ be a polynomial of degree $n$ with complex coefficients. Let

$\operatorname{Re}\left(a_{k}\right)=\alpha_{k}, \operatorname{Im}\left(a_{k}\right)=\beta_{k}$ and a positive number $t$ can be found such that 


$$
\begin{array}{ll}
0 \leq \alpha_{0} \leq t \alpha_{1} \leq t^{2} \alpha_{2} \leq \cdots \cdots \leq t^{k} \alpha_{k} \geq t^{k+1} \alpha_{k+1} \geq \cdots \cdots \geq t^{n} \alpha_{n}>0, & 0 \leq k \leq n \\
0 \leq \beta_{0} \leq t \beta_{1} \leq t^{2} \beta_{2} \leq \cdots \cdots \leq t^{s} \beta_{s} \geq t^{s+1} \beta_{s+1} \geq \cdots \cdots \geq t^{n} \beta_{n}>0, & 0 \leq s \leq n,
\end{array}
$$

then all the zeros of $p(z)$ lie in the disk $|z| \leq \frac{t}{\left|a_{n}\right|}\left\{2\left(\frac{t^{k} \alpha_{k}+t^{s} \beta_{s}}{t^{n}}\right)-\left(\alpha_{n}+\beta_{n}\right)\right\}$

The above result is due to Aziz and Mohammad [1].

The above result does not give zero-free region inside disk and is based upon the assumption that all $a_{j}{ }^{\prime} s$ and $\beta_{j}{ }^{\prime} s$ are positive numbers. We have improved and generalized this result by obtaining a zero-free region inside the disk and also maximum number of zeros in prescribed region. We have also assumed that $a_{j}{ }^{\prime} s$ and $\beta_{j}$ 's may take any negative or positive values. More precisely we prove

Theorem: -Let $p(z)=\sum_{v=0}^{n} a_{v} z^{v}$ be a polynomial of degree $n$. If $a_{j}=\alpha_{j}+i \beta_{j}$ and for some real number $t>0$,

$$
\begin{aligned}
& \alpha_{0} \leq t \alpha_{1} \leq t^{2} \alpha_{2} \leq \cdots \cdots \leq t^{k-1} \alpha_{k-1} \leq t^{k} \alpha_{k} \geq \cdots \cdots \geq t^{n} \alpha_{n}, \\
& \beta_{0} \leq t \beta_{1} \leq t^{2} \beta_{2} \leq \cdots \cdots \leq t^{s-1} \beta_{s-1} \leq t^{s} \beta_{s} \geq \cdots \cdots \geq t^{n} \beta_{n},
\end{aligned}
$$

where $\alpha_{0}$ and $\beta_{0}$ are not simultaneously zero.

Then no zeros lie in $\frac{t^{2}\left|a_{0}\right|}{M_{1}} \geq|z|$ and number of zeros lying in $\frac{t^{2}\left|a_{0}\right|}{M_{1}} \leq|z| \leq \delta t,(0<\delta<1)$ does not exceed

$$
\frac{1}{\log 1 / \delta} \log \left[\frac{t^{n}\left\{\left|a_{n}\right|-\left(\alpha_{n}+\beta_{n}\right)\right\}+\left\{\left|a_{0}\right|-\left(\alpha_{0}+\beta_{0}\right)\right\}+2\left(t^{k} \alpha_{k}+t^{s} \beta_{s}\right)}{\left|a_{0}\right|}\right],
$$

where

$$
M_{1}=t^{n+1}\left\{\left(\left|\alpha_{n}\right|-\alpha_{n}\right)+\left(\left|\beta_{n}\right|-\beta_{n}\right)\right\}+2 t\left(t^{k} \alpha_{k}+t^{s} \beta_{s}\right)-t\left(\alpha_{0}+\beta_{0}\right) .
$$

Corollary: - If in this theorem we take $\alpha_{j}>0$ and $\beta_{j}>0$, then all the zeros of $p(z)$ as per the conditions of theorem $\mathrm{C}$ lie in

$$
|z| \geq \frac{t\left|a_{0}\right|}{2\left(t^{k} \alpha_{k}+t^{s} \beta_{s}\right)-\left(\alpha_{0}+\beta_{0}\right)} .
$$

This result is an improvement of Theorem C.

\section{2-Proof of Theorem}

Proof of the theorem: - Let $F(z)=(t-z) p(z)$

$$
\begin{aligned}
& =(t-z)\left(a_{0}+a_{1} z+a_{2} z^{2}+\cdots \cdots+a_{n} z^{n}\right) \\
F(z) & =t a_{0}+\sum_{j=1}^{n}\left(t a_{j}-a_{j-1}\right) z^{j}-a_{n} z^{n+1}
\end{aligned}
$$

For $|z| \leq t$ 


$$
\begin{aligned}
|F(z)| & \leq t\left|a_{0}\right|+t^{n+1}\left|a_{n}\right|+\sum_{j=1}^{n}\left|t a_{j}-a_{j-1}\right| t^{j} \\
& \leq t\left|a_{0}\right|+t^{n+1}\left|a_{n}\right|+\sum_{j=1}^{n}\left\{\left|t \alpha_{j}-\alpha_{j-1}\right|+\left|t \beta_{j}-\beta_{j-1}\right|\right\} t^{j} \\
& \leq t\left|a_{0}\right|+t^{n+1}\left|a_{n}\right|+\sum_{j=1}^{k}\left|t \alpha_{j}-\alpha_{j-1}\right| t^{j}+\sum_{j=k+1}^{n}\left|t \alpha_{j}-\alpha_{j-1}\right| t^{j}+\sum_{j=1}^{s}\left|t \beta_{j}-\beta_{j-1}\right| t^{j} \\
+\sum_{j=s+1}^{n}\left|t \beta_{j}-\beta_{j-1}\right| t^{j} & \leq t\left|a_{0}\right|+t^{n+1}\left|a_{n}\right|+2 \alpha_{k} t^{k+1}-t \alpha_{0}-t^{n+1} \alpha_{n}+2 \beta_{s} t^{s}-t \beta_{0}-t^{n+1} \beta_{n} \\
& \leq t^{n+1}\left\{\left|a_{n}\right|-\left(\alpha_{n}+\beta_{n}\right)\right\}+t\left\{\left|a_{0}\right|-\left(\alpha_{0}+\beta_{0}\right)\right\}+2 t\left(t^{k} \alpha_{k}+t^{s} \beta_{s}\right) \\
& =M(\text { Let })
\end{aligned}
$$

Further $F(0)=t a_{0} \neq 0$.

Now it is known that $[5, \mathrm{p}-171]$ if $G(z)$ is regular, $G(0) \neq 0$ and $|G(z)| \leq M$ for $|z| \leq R$, then the number of zeros of $G(z)$ in $|z| \leq \delta R,(0<\delta<1)$ does not exceed $\frac{1}{\log 1 / \delta} \log \frac{M}{|G(0)|}$.Applying this fact to $F(z)$, we get the maximum number of zeros of $F(z)$ and hence $p(z)$ that can lie in $|z| \leq \delta t$ as

$$
\frac{1}{\log 1 / \delta} \log \left[\frac{t^{n}\left\{\left|a_{n}\right|-\left(\alpha_{n}+\beta_{n}\right)\right\}+\left\{\left|a_{0}\right|-\left(\alpha_{0}+\beta_{0}\right)\right\}+2\left(t^{k} \alpha_{k}+t^{s} \beta_{s}\right)}{\left|a_{0}\right|}\right]
$$

This proves the first part of the theorem.

Now to show no zeros lie in $|z| \leq \frac{t^{2}\left|a_{0}\right|}{M_{1}}$, we proceed as follows:

$$
\begin{aligned}
F(z) & =(t-z) p(z) \\
& =(t-z)\left(a_{0}+a_{1} z+a_{2} z^{2}+\cdots \cdots+a_{n} z^{n}\right)
\end{aligned}
$$

This implies that

$$
F(z)=t a_{0}-a_{n} z^{n+1}+\sum_{j=1}^{n}\left(t a_{j}-a_{j-1}\right) z^{j}
$$

or

$$
F(z)=t a_{0}+h(z)
$$

where

$$
h(z)=-a_{n} z^{n+1}+\sum_{j=1}^{n}\left(t a_{j}-a_{j-1}\right) z^{j}
$$

Now for $|z|=t$ 


$$
\begin{aligned}
\underset{|z|=t}{\operatorname{Max}}|h(z)| & \leq\left|a_{n}\right| t^{n+1}+\sum_{j=1}^{n}\left|t a_{j}-a_{j-1}\right| t^{j} \\
& \leq\left(\left|\alpha_{n}\right|+\left|\beta_{n}\right|\right) t^{n+1}+\sum_{j=1}^{n}\left\{\left|t \alpha_{j}-\alpha_{j-1}\right|+\left|t \beta_{j}-\beta_{j-1}\right|\right\} t^{j} \\
& \leq\left(\left|\alpha_{n}\right|+\left|\beta_{n}\right|\right) t^{n+1}+\sum_{j=1}^{n}\left|t \alpha_{j}-\alpha_{j-1}\right| t^{j}+\sum_{j=1}^{n}\left|t \beta_{j}-\beta_{j-1}\right| t^{j} \\
& \leq\left(\left|\alpha_{n}\right|+\left|\beta_{n}\right|\right) t^{n+1}+2 \alpha_{k} t^{k+1}-t \alpha_{0}-t^{n+1} \alpha_{n}+2 \beta_{s} t^{s+1}-t \beta_{0}-t^{n+1} \beta_{n} \\
& \leq t^{n+1}\left\{\left(\left|a_{n}\right|-\alpha_{n}\right)+\left(\left|\beta_{n}\right|-\beta_{n}\right)\right\}+2 t\left(t^{k} \alpha_{k}+t^{s} \beta_{s}\right)-t\left(\alpha_{0}+\beta_{0}\right) \\
& =M_{1}(\text { Let })
\end{aligned}
$$

By Schwarz’s lemma

$$
|h(z)| \leq M_{1} \frac{|z|}{t} \quad \text { For }|z| \leq t .
$$

Therefore $F(z)=t a_{0}+h(z)$ implies that

$$
\begin{aligned}
|F(z)| & \geq t\left|a_{0}\right|-|h(z)| \\
& \geq t\left|a_{0}\right|-M_{1} \frac{|z|}{t} \\
& >0
\end{aligned}
$$

if

$$
|z|<\frac{t^{2}\left|a_{0}\right|}{M_{1}} .
$$

This implies that $F(z)$ and hence $p(z)$ does not vanish if

$$
|z|<\frac{t^{2}\left|a_{0}\right|}{M_{1}} .
$$

This proves the desired result.

\section{REFERENCES}

[1] A. Aziz and Q. G. Mohammad, "Zero free regions for polynomials and some generalization of Eneströ m-Kakeya theorem" Canad. Math. Bull. 27(1984), 265-278.

[2] G. Enestr $\ddot{o} \mathrm{~m}$, "Remarque sur un Theoreme relatif aux recines de I' equation $a_{n} x^{n}+\cdots \cdots+a_{0}=0$ outous les coefficients sont reels et positifs" Tohoku Math. J. 18(1920), 34-36.

[3] N. K. Govil and Q. I. Rahman, "On the Eneströ m-Kakeya theorem” Tohoku Math. J. 20(1968), 126-136.

[4] S. Kakeya, "On the limits of the roots of an algebraic equation with positive coefficients" Tohoku Math. J. 2(1912-13) 140-145.

[5] E. C. Titchmarsh, "The theory of function" $2^{\text {nd }}$ Edition, Oxford University Press London (1939). 


\section{AUTHOR's BIOGRAPHY}

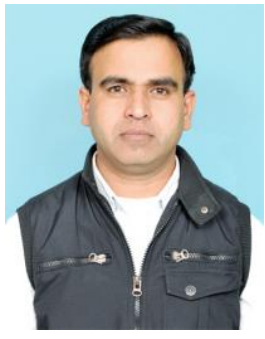

Dr. Roshsn Lal, is an Assistant Professor of Mathematics in Govrnment Degree College, Chaubattakhal, Uttarakhand, India from 11 years. He had his Ph.D. from Jamia Millia Islamia, New Delhi, India. He has membership of Ramanujan Mathematical Society and Indians Mathematical Society. His area of interest is Analysis and Mathematical Modeling. 